\title{
THE QUALITATIVE AND QUANTITATIVE ANALYSIS OF VOLATILE COMPOUNDS OF THE HERB OF DAHLIA NYMPHAEALES OF KEN'S FLAME CULTIVAR
}

\author{
N.I.Ilyinska, T.M.Gontova, Ya.S.Kryukova \\ National University of Pharmacy \\ Key words: sesquiterpenoids; fatty acids; sesquiterpene ethers/essential oils; Dahlia; herbs
}

\begin{abstract}
Many plants consist of volatile substances, these substances possess a variety of pharmacological effects on the human body, the main ones are analgesic, anti-inflammatory, healing, antimicrobial, soothing, and so on. The aim was to study the qualitative composition and quantitative content of volatile substances of the herb of Dahlia Nymphaeales of Ken's Flame cultivar by chromatographymass spectrometry. For the study an Agilent Technology 6890 gas chromatograph with the mass spectrometric detector 5973 was used. As the result of the research 46 substances have been determined, among them 43 substances have been identified. It has been found that sesquiterpinoids a-kadinol, globulol, vulgarol A, spathulenol, allo-spathulenol were dominant among all groups of compounds identified. The quantitative content of this group was $21.9 \%$ of the total amount of compounds. The content of both sesquiterpenoids and fatty acids was the same (17.7\% each). Sesquiterpinoids were represented by 10 compounds: aromadendren, allo-aromadendren, $\beta$-Elemen, $\beta$-farnezen, germacrene $D$, germacrene $B$, shyobunon, farnezen, $\delta$-kadinen, $\beta$-sesquiphellandrene. Shiobunon prevailed among sesquiterpinoids $(210.81 \mathrm{mg} / \mathrm{g})$. The fatty acids were represented by 4 compounds: palmitoleic acid, palmitic acid, oleic acid, linoleic acid, among them palmitoleic acid (200.46 mkg/g) prevailed. The content of triterpenoids ( $\beta$-caryophyllene, $\alpha$-caryophyllene, squalene) was $9.11 \%$. In addition to these compounds the essential oil of Dahlia Nymphaeales herb contained alkanes (8.7\%), sesquiterpene ketones (7.9\%), diterpene alcohols (5\%). Furthermore, the raw material accumulated a trace amount of alkenylphenols (1.6\%), ketones (1.4\%), sesquiterpene oxides, aldehydes and phenols (1.2\% each), monoterpene alcohols (0.32\%).
\end{abstract}

Volatile substances are widespread in the plant world, and possess a number of pharmacological effects such as anti-inflammatory, antibacterial, sedative, analgesic, etc. Essential oils affect the high blood pressure, expand coronary vessels and improve the human sleep [3, 4, 7, 8, 10-12].

Within the systemic analysis of the chemical composition of Dahlia Nymphaeales of Ken's Flame cultivar a number of studies on the qualitative composition of organic acids, amino acids, macro- and microelements, vitamins was carried out $[1,6,11]$. Previous microscopic analysis of the herb of Dahlia Nymphaeales of Ken's Flame cultivar shows that the epidermis of leaves and flowers have the characteristic glandular structures that accumulate essential oils and other volatile compounds [2]. Therefore, it is advisable to study the composition of volatile compounds contained in the herb of Dahlia Nymphaeales of Ken's Flame cultivar.

The aim was to study the qualitative composition and quantitative content of volatile substances in Dahlia Nymphaeales of Ken's Flame cultivar by chromatographymass spectrometry.

\section{Materials and Methods}

For the experiment the herb of Dahlia Nymphaeales of Ken's Flame cultivar was used. It was collected in the flowering period (September, 2013) in Kharkiv region and dried to air-dry state. Volatile compounds in the raw material were identified by chromatography-mass spectrometry. The essential oil was obtained by water distil- lation. For distillation of volatiles $22 \mathrm{ml}$ Agilent vials with open lids and a silicone stopper were used.

Tridecane was used as an internal standard.

According to the procedure $10 \mathrm{ml}$ of water was added to the sample, and distillation of volatile substances with water vapour was conducted for $2 \mathrm{~h}$ under reflux. The substances adsorbed after cooling the system were washed by adding $3 \mathrm{ml}$ of especially pure pentane into a dry $10 \mathrm{ml}$ vial. Washouts were concentrated by blowing $(100 \mathrm{ml} / \mathrm{min})$ of especially pure nitrogen to the residual volume of the extract $(10 \mathrm{mcl})$ completely collected with the chromatographic syringe. Further concentration of the sample was conducted in the syringe to the volume of $2 \mathrm{mcl}$.

The composition of volatile substances was studied on an Agilent Technology 6890 gas chromatograph with the mass spectrometric detector 5973. For the analysis the INNOWAX chromatographic capillary column (the column length $-30 \mathrm{~m}$, the internal diameter $-0.25 \mathrm{~mm}$ ) was used, the carrier gas was helium with the speed of $1.2 \mathrm{ml} / \mathrm{min}$. The introduction of the sample was carried with separation of the flow $1 / 50$. The thermostat temperature was $50^{\circ} \mathrm{C}$ to $220^{\circ} \mathrm{C}$ at the rate of $4 \% \mathrm{~min}$. The temperature of the detector and the evaporator was $250^{\circ} \mathrm{C}$. The sample was injected in a splitless mode (without the flow separation) to the chromatographic column $(2 \mathrm{mcl})$, the rate of the sample introduction was $1.2 \mathrm{ml} / \mathrm{min}$ for $0.2 \mathrm{~min}$. To identify the components the libraries of 
Table NISTO5 and WILEY 2007 mass spectra with the total

The components of volatile compounds obtained from the herb of Dahlia Nymphaeales of Ken's Flame cultivar

\begin{tabular}{|l|c|c|}
\hline \multicolumn{1}{|c|}{ Compound } & $\begin{array}{c}\text { Retention time, } \\
\text { min }\end{array}$ & $\begin{array}{c}\text { Content } \\
\mathrm{mkg} / \mathrm{g}\end{array}$ \\
\hline nonanal & 9.69 & 1.93 \\
\hline linalool & 9.77 & 4.04 \\
\hline terpineol & 12.50 & 2.66 \\
\hline decanal & 12.99 & 4.82 \\
\hline 3-methoxyacetophenone & 16.21 & 6.41 \\
\hline methyl-trans-eugenol & 17.63 & 3.81 \\
\hline$\beta$-elemen & 19.28 & 4.91 \\
\hline$\beta$-caryophyllene & 20.05 & 19.82 \\
\hline cis-methyl eugenol & 20.62 & 31.20 \\
\hline allo-aromadendrene & 20.94 & 1.58 \\
\hline a-caryophyllene & 21.04 & 5.43 \\
\hline$\beta$-farnesene & 21.30 & 5.37 \\
\hline germacrene D & 21.86 & 40.84 \\
\hline germacrene B & 22.32 & 15.21 \\
\hline shyobunon & 22.70 & 210.81 \\
\hline farnesene & 22.81 & 5.98 \\
\hline$\delta$-cadinene & 23.13 & 9.12 \\
\hline$\beta$-sesquiphellandrene & 23.21 & 5.13 \\
\hline caryophyllene oxide & 23.85 & 25.43 \\
\hline allo-spathulenol & 24.53 & 22.82 \\
\hline spathulenol & 24.70 & 279.26 \\
\hline globulol & 24.82 & 50.87 \\
\hline aromadendren & 26.10 & 75.35 \\
\hline a-cadinol & 26.76 & 94.10 \\
\hline azulenon & 27.20 & 106.47 \\
\hline 1,4 -cis-1,7-trans-akorenon & 27.34 & 59.49 \\
\hline 2-methoxy-5-vinylphenol & 27.88 & 33.06 \\
\hline tetradecanal & 27.98 & 17.08 \\
\hline not identified & 28.13 & 20.87 \\
\hline not identified & 28.95 & 47.05 \\
\hline vulgarol A & 29.04 & 14.79 \\
\hline farnesyl acetone & 31.21 & 23.52 \\
\hline palmitoleic acid & 31.76 & 47.13 \\
\hline palmitic acid & 32.17 & 200.46 \\
\hline octadecanal & 33.80 & 2.49 \\
\hline phytol & 34.11 & 106.68 \\
\hline oleic acid & 34.30 & 101.05 \\
\hline not identified & 34.42 & 27.55 \\
\hline linoleic acid & 34.46 & 24.44 \\
\hline tricosane & 36.94 & 92.03 \\
\hline tetracosane & & 11.89 \\
\hline pentacosane & 39.17 & 15.37 \\
\hline hexacosane & 5.75 \\
\hline heptacosane & 24.69 \\
\hline squalene & 33.97 \\
\hline nonacosane & \\
\hline
\end{tabular}

number of more than 470000 spectra were used along with AMDIS and NIST identification programmes [5,9].

\section{Results and Discussion}

According to the data of the chromatography-mass spectrometry study 46 compounds have been found in the herb of Dahlia Nymphaeales of Ken's Flame cultivar. Among them 43 substances have been identified (Table).

Among the groups of compounds identified by the quantitative content sesquiterpenoids prevailed (21.9\% of the total volatile compounds); they were represented by $\alpha$-kadinol, vulgarol A globulol, spathulenol, allospathulenol with spathulenol being a dominant compound among sesquiterpenoids $(279.26 \mathrm{mkg} / \mathrm{g})$. The content of both sesquiterpenoids and fatty acids was the same $(17.7 \%$ each). Sesquiterpenoids were represented by $10 \mathrm{com}$ pounds: aromadendren, allo-aromadendren, $\beta$-elemen, $\beta$-farnesene, germacrene $\mathrm{D}$, germacrene $\mathrm{B}$, shiobunon, farnesene, $\delta$-cadinene, $\beta$-sesquiphellandrene with shiobunon being a dominant compound among sesquiterpenoids $(210.81 \mathrm{mkg} / \mathrm{g})$. Among the fatty acids (palmitoleic, palmitic, oleic, linolenic) the largest amount was registered for palmitoleic acid $(200.46 \mathrm{mg} / \mathrm{g})$.

The compounds of the triterpene group were represented by $\beta$-caryophyllene, $\alpha$-caryophyllene, squalene. The total amount of these substances was $9.11 \%$. Among triterpenes squalene prevailed (167.07\%). In addition to these compounds the essential oil of Dahlia Nymphaeales herb contained alkanes $(8.7 \%$ - tricosane, tetracosane, pentakosane, heptacosane, hexacosane, nonacosane), sesquiterpene ketones $(7.9 \%$ - azulenon, 1,4-cis-1,7-trans-akorenon), diterpene alcohols (5\% phytol), alkylphenols $(1.6 \%$ - 2-methoxy-5-vinylphenol). Ketones - farnesylacetone, 3-methoxyacetophenone amounted to $1.4 \%$.

The amount of phenols (methyl-trans-eugenol, cismethyleugenol), aldehydes (nonanal, decanal, tetradecanal, octadecanal) and sesquiterpene oxides (caryophyllene oxide) in Dahlia herb was equal $-1.2 \%$ each The content of monoterpene alcohols (linalool, terpineol) in Dahlia herb was in trace amount $(0.32 \%)$.

\section{CONCLUSIONS}

1. For the first time chromatography-mass spectrometry has been applied to study the qualitative composition and quantitative content of volatile compounds in the herb of Dahlia Nymphaeales of Ken's Flame cultivar. Among them 43 substances have been identified, and their quantitative content has been determined.

2 . The dominant groups of compounds were sesquiterpene alcohols ( $21.9 \%$ of the total amount of volatile compounds), with spathulenol being the largest in quantity $(279.26 \mathrm{mkg} / \mathrm{g})$; fatty acids $(17.7 \%)$, with palmitoleic acid exceeding the rest $(200.46 \mathrm{mkg} / \mathrm{g})$ and sesquiterpenoids $(17.7 \%)$, with shyobunon being the largest in quantity $(210.81 \mathrm{mkg} / \mathrm{g})$. 


\section{REFERENCES}

1. Гонтова Т.М., Кічимасова Я.С., Ільӥнська Н.І. // Зб. наук. праџьь співробіт. НМАПО ім. П.Л.Шупика. 2014. - Bun. 23 (4). - C. 245-249.

2. Плахотнича С.А., Ільїнська Н.І., Кічимасова Я.С. // Досягнення сучасної медицини: матер. 75-ї загальноуніверситетської студ. наук. конф., м. Львів 24-25 квітня 2014 р. - Л., 2014. - С. 222-223.

3. Astani A., Reichling J., Schnitzler P. // Phytotherapy Res. - 2010. - Vol. 24 (5). - P. 673-679.

4. Bassole I., Juliani H.R. // Molecules. - 2012. - Vol. 17 (4). - P. 3989-4006.

5. Bicchi C., Brunelli C., Cordero C. // J. Chromatogr. A. - 2004. - Vol. 1024, №1-2. - P. 195-207.

6. Ilyinska N., Gontova T., Kichymasova Y. // Actual Questions of Development of New Drugs: XX International scientific and practical conference of young scientists and student, April 22-23, 2014, Kharkiv. - P. 32.

7. Khan M.A. Chervinskaya A.V., Sotnikova E.N. // Problems of Balneol., Physiotherapy and Exercise Therapy. - 2005. - Vol. 2. - P. 9-12.

8. Kovaleva A.M., Goncharov N.F., Komissarenko A.N. et al. // Chemistry of Natural Compounds. - 2009. - Vol. 45, №4. - P. 592-593.

9. Roman M., Dobrowolski J.C., Baranska M. et al. // J. of Natural Products. - 2011. - Vol. 74 (8). - P. $1757-1763$.

10. Sacchetti G., Maietti S., Muzzoli M. et al. // Food Chemistry. - 2005. - Vol. 91 (4). - P. 621-632.

11. Sokolova O., Gontova T., Ilyinska N., Matkowski A. // Trends in natural products research: young scientists meeting, June 23-25, 2014, Olomouc. - P. 58-59.

12. Weitzel C. // Phytochemistry. - 2011. - Vol. 72. - P. 572-578.

\section{ЯКІСНИЙ СКЛАД ТА КІЛЬКІСНИЙ ВМІСТ ЛЕТКИХ СПОЛУК ТРАВИ ЖОРЖИНИ НІМФЕЙНОЇ COPTY KEN'S FLAME \\ Н.І.Ільїнська, Т.М.Гонтова, Я.С.Крюкова}

Ключові слова: сесквітерпенові спирти; сесквітерпеноїди; жирні кислоти; жоржина; трава Леткі речовини у значній мірі входять до складу багатьох рослин і чинять різноманітні фрармакологічні ефекти на організм людини, основними з яких є знеболювальна, протизапальна, ранозагоювальна, антимікробна, заспокійлива дія тощо. Метою роботи стало вивчення якісного складу та кількісного вмісту летких речовин трави жоржини німфейної copmу Ken's Flame методом хромато-мас-спектрометрії. Для дослідження використовували газовий хромamoгpaфp Agilent Technology 6890 із мас-спектрометричним детектором 5973. У результаті проведених досліджень виявлено 46 речовин, з яких ідентифіковано 43. Встановлено, що серед ідентифрікованих груп сполук домінували сесквітерпенові спирти: $\alpha$-кадинол, глобулол, вульгарол А, спатуленол, ало-спатуленол. Кількісний вміст иієї групи речовин становив 21,9\% від загальної кількості сполук. Вміст сесквітерпеноїдів і жирних кислот був однаковим і складав по 17,7\%. Сесквітерпеноїди представлені 10 сполуками: аромадендреном, ало-аромадендреном, $\beta$-елеменом, $\beta$-фрарнезеном, гермакреном Д, гермакреном $B$, шиобуноном, фрарнезеном, $\delta$-кадиненом, $\beta$-сесквіфріландреном, серед яких переважав шиобунон (210,81 мка/2). Жирні кислоти представлені 4 сполуками: пальмітолеїновою, пальмітиновою, олеїновою, ліноленовою кислотами, з яких у більшій кількості містилася пальмітинова кислота (200,46 мкг/2). Вміст тритерпеноїдів (ß-каріофілен, а-каріофілен, сквален) склав 9,11\%. Окрім перелічених вище груп сполук у траві жоржини німфейної встановлено наявність алканів (8,7\%), сесквітерпенових кетонів (7,9\%), дитерперпенових спиртів (5\%). Крім того, у незначній кількості у досліджуваній сировині накопичувались алкенілфеноли (1,6\%), кетони $(1,4 \%)$, сесквітерпенові оксиди, френоли та альдегіди (по 1,2\%), монотерпенові спирти (0,32\%).

\section{КАЧЕСТВЕННЫЙ СОСТАВ И КОЛИЧЕСТВЕННОЕ СОДЕРЖАНИЕ ЛЕТУЧИХ СОЕДИНЕНИЙ ТРАВЫ ГЕОРГИНЫ НИМФЕЙНОЙ СОРТА KEN'S FLAMЕ Н.И.Ильинская, Т.Н.Гонтовая, Я.С.Крюкова}

Ключевые слова: сесквитерпеновые спирты; сесквитерпеноиды; жирные кислоты; георгина; трава

Летучие вещества в значительной степени входят в состав многих растений и обладают различными фрармакологическими эффректами на организм человека, основными из которых являются обезболивающее, противовоспалительное, ранозаживляющее, антимикробное, успокаивающее действие и т. д. Целью работы стало изучение качественного состава и количественного содержания летучих веществ травы георгины нимфейной сорта Ken's Flame методом хромато-масс-спектрометрии. Для исследования использовали газовый хромаmoгpadp Agilent Technology 6890 с масс-спектрометрическим детектором 5973. В результате проведенных исследований обнаружено 46 веществ, из которых идентифицировано 43. Установлено, что среди идентифицированных групп соединений доминировали сесквитерпе-

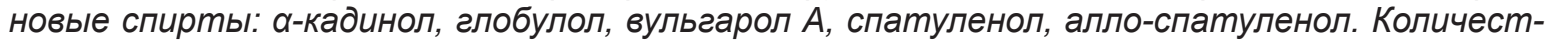


венное содержание этой группы веществ составило 21,9\% от общего количества соединений. Содержание сесквитерпеноидов и жирных кислот было одинаковым и составило по 17,7\%. Сесквитерпеноиды представлены 10 соединениями: аромадендреном, алло-аромадендреном, $\beta$-элеменом, $\beta$-фрарнезеном, гермакреном Д, гермакреном $B$, шиобуноном, фрарнезеном, б-кадиненом, $\beta$-сесквифриландреном, среди которых преобладал шиобунон (210,81 мкг/2). Жирные кислоты представлены 4 соединениями: пальмитолеиновой, пальмитиновой, олеиновой, линоленовой кислотами, из которых в большем количестве содержалась пальмитиновая кислота (200,46 мкг/2). Содержание тритерпеноидов ( $\beta$-кариофиллен, $\alpha$-кариофиллен, сквален) составило 9,11\%. Кроме перечисленных выше групп соединений в траве георгины нимфрейной установлено наличие алканов (8,7\%), сесквитерпеновых кетонов (7,9\%), дитерперпеновых спиртов (5\%). Кроме того, в незначительном количестве в исследуемом сырье накапливались алкенилфенолы (1,6\%), кетоны (1,4\%), сесквитерпеновые оксиды, френолы и альдегиды (по 1,2\%), монотерпеновые спирты (0,32\%). 